\title{
Combination of Inferior Vena Cava Diameter, Hepatic Venous Flow, and Portal Vein Pulsatility Index: Venous Excess Ultrasound Score (VEXUS Score) in Predicting Acute Kidney Injury in Patients with Cardiorenal Syndrome: A Prospective Cohort Study
}

Vimal Bhardwaj ${ }^{1}$, Gunaseelan Vikneswaran ${ }^{2}$, Philippe Rola ${ }^{3}$, Siddharth Raju ${ }^{4}$, Rammohan S Bhat ${ }^{5}$, Arunkumar Jayakumar ${ }^{6}$, Arjun Alva $^{7}$

\begin{abstract}
Background: Fluid overload is deleterious in critically ill patients. It can lead to venous congestion, thereby increasing venous pressure, theoretically increasing the backpressure, and thereby reducing renal blood flow. Venous congestion thus can be an important contributor to acute kidney injury (AKI), with no validated tools to objectively identify venous congestion bedside.

Materials and methods: Patients above 18 years admitted in ICU with a provisional diagnosis of cardiorenal syndrome were included in the study. Those with inadequate window, inferior vena cava (IVC) thrombus, and known case of cirrhosis with portal hypertension were excluded from the study. Patients underwent ultrasound examination with serial determination till AKI resolved or patient is initiated on dialysis. Venous excess ultrasound score (VEXUS) comprising inferior vena cava, hepatic vein waveform, and portal vein pulsatility was assessed.

Results: Thirty patients were enrolled for the study. The mean age was $59.53 \pm 16.47$ with $21(70 \%)$ males. Mean sequential organ failure assessment (SOFA) score was $5.03 \pm 1.97$. Fourteen patients (46.7\%) were in AKI stage 1, while eight patients (26.7\%) were in AKI stage 2 and stage 3 each. Twenty patients (66.7\%) had VEXUS grade III. Resolution of AKI injury showed significant correlation with improvement in VEXUS grade ( $p$ value 0.003). Similarly, there was significant association between changes in VEXUS grade and fluid balance ( $p$ value 0.006$)$. There was no correlation between central venous pressure (CVP), left ventricular function, and right ventricular function with change in VEXUS grade.

Conclusion: The study shows that a combined grading of IVC, hepatic vein, and portal vein might reliably demonstrate venous congestion and aid in the clinical decision to perform fluid removal.

Keywords: Acute kidney injury, Fluid balance, Portal vein pulsatility, Venous congestion, Volume overload.

Indian Journal of Critical Care Medicine (2020): 10.5005/jp-journals-10071-23570
\end{abstract}

\section{BACKGROUND}

Fluid overload is deleterious in critically ill patients; apart from increased mortality, it can cause end-organ damage, thereby increasing the incidence of acute kidney injury (AKI), length of stay in ICU, and duration of mechanical ventilation. ${ }^{1-3}$ The attributable mortality to $\mathrm{AKI}$ is $20 \%$ and is an independent predictor of mortality. ${ }^{4}$

Fluid overload leads to venous congestion, thereby increasing venous pressure and theoretically increasing the backpressure and reducing renal blood flow, and since kidneys are encapsulated organs it might make them more sensitive to this effect.

There is no clear consensus to measure renal venous pressure. Clinical signs of fluid overload like peripheral edema and ascites are poor indicators of fluid overload in the ICU setup. ${ }^{5}$ Central venous pressure (CVP), which is routinely used in ICU, has shown to be associated with increased incidence of AKI, but CVP as a single measure reflecting venous pressure is highly questionable as there is no clear consensus on reference value, position of the patients, effect of varying intrathoracic pressure in mechanically ventilated patients, and might not reflect preload directly. ${ }^{6,7}$

In view of lack of reliable peripheral signs of venous congestion, objective determination of the same is the need of the hour to aid key clinical decisions like stopping fluids, diuretic administration, and management of $\mathrm{AKI}$, to name a few. The underlying
${ }^{1,7}$ Department of Critical Care Medicine, Mazumdar Shaw Medical Center, Narayana Health City, Bengaluru, Karnataka, India

${ }^{2}$ Department of Clinical Research, Narayana Hrudayalaya, Bengaluru, Karnataka, India

${ }^{3}$ Intensive Care Unit Services, Santa Cabrini Hospital, Montreal, Canada

${ }^{4}$ Department of Critical Care Medicine, Narayana Health City, Bengaluru, Karnataka, India

${ }^{5,6}$ Department of Nephrology, Narayana Health City, Bengaluru, Karnataka, India

Corresponding Author: Vimal Bhardwaj, Department of Critical Care Medicine, Mazumdar Shaw Medical Center, Narayana Health City, Bengaluru, Karnataka, India, Phone: +91 9686124830, e-mail: vmlbhardwaj@yahoo.co.in

How to cite this article: Bhardwaj V, Vikneswaran G, Rola P, Raju S, Bhat RS, Jayakumar A, et al. Combination of Inferior Vena Cava Diameter, Hepatic Venous Flow, and Portal Vein Pulsatility Index: Venous Excess Ultrasound Score (VEXUS Score) in Predicting Acute Kidney Injury in Patients with Cardiorenal Syndrome: A Prospective Cohort Study. Indian J Crit Care Med 2020;24(9): 783-789.

Source of support: Nil

Conflict of interest: None

(c) The Author(s). 2020 Open Access This article is distributed under the terms of the Creative Commons Attribution 4.0 International License (https://creativecommons. org/licenses/by-nc/4.0/), which permits unrestricted use, distribution, and non-commercial reproduction in any medium, provided you give appropriate credit to the original author(s) and the source, provide a link to the Creative Commons license, and indicate if changes were made. The Creative Commons Public Domain Dedication waiver (http://creativecommons.org/publicdomain/zero/1.0/) applies to the data made available in this article, unless otherwise stated. 
physiological principle has been considered to grade venous congestion bedside.

The inferior vena cava (IVC) is the initial screen for venous congestion. Whether due to increased stressed venous volume or an increased right atrial pressure (for any given reason), the first venous compartment where congestion becomes apparent is the IVC. In an observational trial by Guinot et al., right ventricular systolic dysfunction and venacaval dilatation preceded altered renal function in postoperative cardiac surgery patients serving as a marker of venous congestion. ${ }^{8}$ Dilated inferior vena cava was associated with renal failure but it was found to be inconsistent as a marker for hemodynamic congestion. ${ }^{9}$

When the IVC reaches the flat part of its compliance, the pressure begins to be transmitted in a retrograde fashion and dilation of the hepatic venous tree is seen and so on for the renal veins. The portal system may show abnormalities as the congestion becomes more severe, making it potentially a more specific marker of an injurious level of congestion. This likely takes place because the pressure has to transmit across the hepatic sinusoids, which buffer the hepatic venous pressure. Eventually, the portal venous flow pattern changes, going from a monophasic signal to a progressively more pulsatile pattern that can eventually become interrupted (100\% pulsatility). A fairly linear relationship between right atrial pressure and the portal vein pulsatility index has been described in congestive heart failure (CHF) patients in acute exacerbation. ${ }^{10} \mathrm{~A}$ case series published by Denault et al. on post-cardiac surgery patients showed portal vein Doppler assessment to be a promising modality for end-organ venous congestion in post-cardiac surgery patients. ${ }^{11}$ Portal vein is subjected to variation because of body habitus and intrathoracic pressure. $^{12,13}$

Hence, venous congestion leads to distension of IVC followed by hepatic venous flow abnormality along with development of portal vein pulsatility and renal venous Doppler flow abnormalities, which was proposed as the VEXUS scoring system. ${ }^{10}$ By utilizing multiple parameters, the negative aspects of individual parameters might get negated and emerge as a reliable tool to assess congestion of kidneys.

There has been a greater emphasis on maintaining the perfusion than identifying venous congestion, which can be an important contributor to $\mathrm{AKl}$; there are no validated tools to objectively identify venous congestion bedside, hence we hypothesize that venous excess ultrasound score (VEXUS), which uses three parameters, i.e., inferior vena cava, hepatic vein waveform, and portal vein pulsatility, correlates with severity of fluid overload. In our study, we have not included renal venous Doppler, which was proposed in the original VEXUS score.

\section{Materials and Methods}

The single-center prospective study was conducted at medical ICU and recruited patients admitted with provisional diagnosis of cardiorenal syndrome during a period of 6 months from September 2019 to February 2020. Patients aged more than 18 years being admitted to ICU with a provisional diagnosis of cardiorenal syndrome were eligible for the study. Patients were excluded from study if they were known cases of cirrhosis with portal hypertension, not willing for ultrasound examination, inadequate window, and IVC thrombus. Patients enrolled in the study underwent ultrasound examination with serial determination till AKI resolved or patient is initiated on dialysis. The study was approved by the institutional ethics committee. The primary objective was to assess the correlation between serial VEXUS score and AKI in patients with cardiorenal syndrome. The secondary objective was to assess correlation between VEXUS score and fluid balance, right heart function, and clinical signs of fluid overload.

\section{Data Collection}

Demographic details along with clinical diagnosis and AKI staging done were noted. Creatinine measurements with urine output, daily fluid balance and sequential organ failure assessment (SOFA) score, CVP, MAP, vasopressors use, if any, use of mechanical/noninvasive mechanical ventilation, baseline echocardiographic recording of ejection fraction, pulmonary artery pressure, TAPSE, use of nephrotoxic agents, and contrast administration were documented on a daily basis. Serial digital images of the deidentified patients' data were recorded and maintained in a digital library. Daily VEXUS score along with AKI staging was done for all the enrolled patients.

Acute kidney injury was defined by the AKIN criteria and staging was done according to the AKIN criteria. The AKIN classification was used in the study; an increase in serum creatinine of more than $0.3 \mathrm{mg} / \mathrm{dL}$ or a decrease in urine output within 48 hours will be considered as AKI. ${ }^{14,15}$

\section{Ultrasound Assessment}

Ultrasound assessment was performed bedside using Sonosite M Turbo machine using a curvilinear abdominal probe of $8-3 \mathrm{MHz}$ by intensivist who is experienced in ultrasound examination for at least 3 years. The investigator performing the ultrasound examination was blinded to serum creatinine values. Patients were positioned in the dorsal decubitus position with headend of bed elevation between $0^{\circ}$ and $30^{\circ}$.

Hepatic Doppler visualizing either the middle hepatic vein in the subxiphoid area or the right hepatic vein from a lateral angle was done. Similarly, the portal vein Doppler was interrogated from a lateral approach. As for all Doppler examinations, the waveforms were traced during a respiratory pause, if respiratory liver movement prevented proper trace generation.

The IVC is interrogated in long and short axis along the intrahepatic segment and a visual average is done. Respiratory variation was defined as a $20 \%$ or more change in surface area in the short axis.

Grade 0: $<5 \mathrm{~mm}$ with respiratory variation

Grade I: 5-9 $\mathrm{mm}$ with respiratory variation

Grade II: $10-19 \mathrm{~mm}$ with respiratory variation

Grade III: $>20 \mathrm{~mm}$ with respiratory variation

Grade IV: $>20 \mathrm{~mm}$ with minimal or no respiratory variation

Hepatic vein (HV): interrogation by pulsed wave Doppler, identification and analysis of $A, S$, and $D$ waves:

Grade 0: normal S > D

Grade I: $S<D$ with antegrade $S$

Grade III: $S$ flat or inverted or biphasic trace

We have chosen the approach that the $S$ wave is normally the larger of the two negative deflections.

Portal vein Doppler (PD)

Portal vein (PV) interrogation

Grade 0: $<0.3$ pulsatility index

Grade I: $0.3-0.49$ pulsatility index

Grade III: $0.5-1.0$ pulsatility index

Pulsatility index is calculated as $\left(V_{\max }-V_{\min }\right) / V_{\max }$

The individual Doppler staging has been compiled into VEXUS staging of venous congestion. 
Venous Excess Ultrasound Score (VEXUS)

Grade 0: IVC grade <III, HD grade 0, PV grade 0

Grade I: IVC grade IV, but normal HV/PV pattern

Grade II: IVC grade IV with mild flow pattern abnormalities in HV/PV

Grade III: IVC grade IV with severe flow pattern abnormalities in $\mathrm{HV} / \mathrm{PV}$

\section{Statistical Analysis}

Data were analyzed using the SPSS software version 19. Continuous variables were expressed using mean and standard deviation. Categorical variables were expressed using frequency and percentage. Patients were categorized into three groups (VEXUS score improving, no change, and worsening) based on change in VEXUS score from baseline. Association between change in VEXUS score and improvement in AKI grading was analyzed by the Fischer exact test. For secondary objective, the Fischer exact test was used to test for association between VEXUS score and signs of fluid balance, right heart function, and clinical signs of fluid overload. The Kruskal-Wallis test used to compare fluid balance between the three categories of VEXUS score followed by the Bonferroni test for between group comparison. The $p$ value less than 0.05 was considered statistically significant.

\section{Results}

A total of 35 patients were enrolled into the study, and 5 patients were excluded in view of poor visualization of portal and hepatic vein.

Thirty patients with AKI were included for the study with a mean age of $59.53 \pm 16.47$. Seventy percent of them ( 21 of 30 ) were males. Mean SOFA score was $5.03 \pm 1.97$. Fourteen patients (46.7\%) were in AKI stage 1, while eight patients (26.7\%) were in AKI stage 2 and stage 3 each. Twenty patients (66.7\%) had a VEXUS grade III (Table 1).

Patients were categorized on day 3 based on VEXUS grade as improving, no change, or worsening. Resolution of AKI injury showed significant correlation with improvement in the VEXUS grade ( $p$ value 0.003 ). Similarly, there was significant association between changes in the VEXUS grade and fluid balance ( $p$ value 0.006). There was no correlation between CVP, left ventricular function, and right ventricular function with change in VEXUS grade (Table 2). There was a significant difference in fluid balance between the VEXUS score improving group $(0.20 \pm 1.24)$ compared to the no change group $(1.67 \pm 1.03)$ and the worsening group $(1.00 \pm 0.00)$ ( $p$ value 0.03 ).

Figure 1 shows relationship between the VEXUS grade and AKI staging. With improvement in kidney function, there is decline in the VEXUS grade as well.

Patients were assessed for the presence of pulmonary edema, pleural effusion, pedal edema, and ascites and were categorized based on the number of positive signs. Peripheral signs of volume overload were not associated with change in the VEXUS grade (Table 3). There was no association with use of IV fluids, inotropes, diuretics, and dialysis on change in VEXUS grades (Table 4).

\section{Discussion}

Acute kidney injury is characterized by a rapid deterioration of kidney function; its occurrence in the ICU is associated with significant mortality and morbidity with an attributable mortality of $40-65 \%$. $^{1}$
Table 1: Baseline characteristics of study subjects

\begin{tabular}{|c|c|}
\hline Variable & Description $(n=30)$ \\
\hline Age (years) & $59.53 \pm 16.47$ \\
\hline \multicolumn{2}{|l|}{ Gender } \\
\hline Male (\%) & $21(70 \%)$ \\
\hline \multicolumn{2}{|l|}{ Diagnosis } \\
\hline Cor pulmonale with AKI & $8(26.66 \%)$ \\
\hline Decompensated heart failure with AKI & $17(56.66 \%)$ \\
\hline Myocarditis with AKI & $5(16.66 \%)$ \\
\hline Central venous pressure & $18.20 \pm 4.75$ \\
\hline \multicolumn{2}{|l|}{ AKIN stage } \\
\hline Stage 1 & $14(46.7 \%)$ \\
\hline Stage 2 & $8(26.7 \%)$ \\
\hline Stage 3 & $8(26.7 \%)$ \\
\hline \multicolumn{2}{|l|}{ VEXUS score } \\
\hline 0 & $3(10 \%)$ \\
\hline 1 & $2(6.7 \%)$ \\
\hline 2 & $5(16.7 \%)$ \\
\hline 3 & $20(66.7 \%)$ \\
\hline SOFA score & $5.03 \pm 1.97$ \\
\hline Ascitis & $15(50 \%)$ \\
\hline Pulmonary edema & $14(46.7 \%)$ \\
\hline Pedal edema & $29(96.7 \%)$ \\
\hline Pleural effusion & $13(43.3 \%)$ \\
\hline Sepsis & $5(16.7 \%)$ \\
\hline Mechanical ventilation & $23(76.7 \%)$ \\
\hline Vasopressors & $6(20 \%)$ \\
\hline \multicolumn{2}{|l|}{ Cardiac function } \\
\hline Ejection fraction $\%$ & $42.50 \pm 10.06$ \\
\hline $\begin{array}{l}\text { Pulmonary artery systolic pressure (PASP) } \\
\mathrm{mm} \mathrm{Hg}\end{array}$ & $45.63 \pm 12.10$ \\
\hline
\end{tabular}

Continuous variables expressed as mean and standard deviation. Categorical variables expressed in frequency and percentage. AKI, acute kidney injury

Acute kidney injury can be caused by multiple factors, i.e., circulatory failure, sepsis, nephrotoxic agents, vasopressors, as well as venous congestion. There has long been an emphasis on forward flow, with a focus on maintaining MAP, avoiding nephrotoxic agents, as well as the aggressive treatment of sepsis. However, the quest to achieve normovolemia and normotension with aggressive fluid therapy and vasopressors may be counterproductive as it can lead to fluid overload and venous congestion leading to a decrease in organ perfusion. Indeed, the focus, traditionally being on managing the inflow pressure (MAP), the outflow pressure, a critical determinant of the organ perfusion pressure, is generally overlooked in clinical practice, with important consequences (Figs 2 to 6).

Our study uses a new scoring system: the VEXUS for the evaluation of venous congestion in a cohort of patients with cardiorenal syndrome. As AKI has many confounding factors, we chose cardiorenal syndrome patients as they are more prone to venous congestion. The study tests the utility of bedside serial ultrasonographic examination of two-dimensional and spectral Doppler imaging of the inferior vena cava, hepatic vein, and portal vein, with a derived scoring system based on hypothesis that the 
Table 2: Comparison of change in kidney function and fluid balance with change in VEXUS score

\begin{tabular}{|c|c|c|c|c|}
\hline & $\begin{array}{l}\text { VEXUS score } \\
\text { improving }\end{array}$ & $\begin{array}{l}\text { VEXUS score } \\
\text { no change }\end{array}$ & $\begin{array}{l}\text { VEXUS score } \\
\text { worsening }\end{array}$ & $p$ value \\
\hline AKI & & & & $0.003^{*}$ \\
\hline Resolving & $20(87 \%)$ & $3(13 \%)$ & 0 & \\
\hline Not resolving & $2(28.6 \%)$ & $3(42.9 \%)$ & $2(28.6 \%)$ & \\
\hline \multicolumn{5}{|l|}{ Fluid balance as } \\
\hline Decreasing & 17 (89.5\%) & 2 (10.5\%) & 0 & $0.006^{*}$ \\
\hline Increasing & $3(33.3 \%)$ & $4(44.4 \%)$ & $2(22.2 \%)$ & \\
\hline $\begin{array}{l}\text { Fluid balance in } \\
\text { liters day } 2\end{array}$ & $0.20 \pm 1.24$ & $1.67 \pm 1.03$ & $1.00 \pm 0.00$ & 0.03 \\
\hline CVP & & & & 0.15 \\
\hline Normalized & 8 (88.9\%) & 0 & $1(11.1 \%)$ & \\
\hline $\begin{array}{l}\text { Not } \\
\text { normalized }\end{array}$ & $12(63.2 \%)$ & $6(31.6 \%)$ & $1(5.3 \%)$ & \\
\hline LVF & & & & 0.65 \\
\hline Normal & 15 (78.9\%) & $3(15.8 \%)$ & $1(5.3 \%)$ & \\
\hline Reduced & $7(63.6 \%)$ & $3(27.3 \%)$ & $1(9.1 \%)$ & \\
\hline RVF & & & & 0.19 \\
\hline Normal & $16(84.2 \%)$ & 2 (10.5\%) & $1(5.3 \%)$ & \\
\hline Reduced & $6(54.5 \%)$ & $4(36.4 \%)$ & $1(9.1 \%)$ & \\
\hline
\end{tabular}

Statistical test used the Fischer exact test for categorical outcomes and the Kruskal-Wallis test for fluid balance in liters. ${ }^{*} p$ value less than 0.05 considered statistically significant. CVP, central venous pressure; LVF, left ventricular function; RVF, right ventricular function

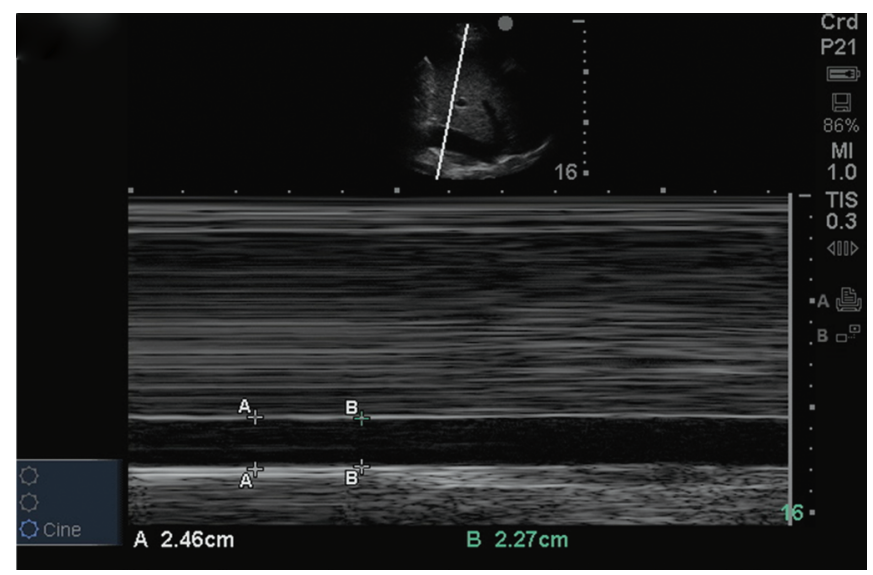

Fig. 1: Intrahepatic portion of IVC with no respiratory variation (grade IV IVC according to staging)

first venous compartment where congestion becomes apparent is the IVC. When the IVC reaches the flat part of its compliance, the pressure begins to be transmitted in a retrograde fashion and dilation of the hepatic venous tree is seen followed by the development of portal vein pulsatility and renal venous Doppler flow abnormalities. This scoring system was first validated by Beaubien-Souligny et al. in postoperative cardiac surgery patients where a significant association was found between an elevated VEXUS score and the development of AKI. ${ }^{16}$

Our study results support the hypothesis that serial VEXUS scoring is correlated with AKI staging. Among 30 patients, 20 patients with resolving AKI correlated with the downward/ improving trend of VEXUS score accounting for $87 \%$ of cases associated with a decreasing trend of fluid balance. Five patients
Table 3: Association of VEXUS score with signs of volume load

\begin{tabular}{lllll}
\hline Peripheral signs & $\begin{array}{l}\text { VEXUS score } \\
\text { improving }\end{array}$ & $\begin{array}{l}\text { VEXUS score } \\
\text { no change }\end{array}$ & $\begin{array}{l}\text { VEXUS score } \\
\text { worsening }\end{array}$ & p value \\
\hline $\begin{array}{l}\text { One sign } \\
\text { positive }\end{array}$ & $7(70 \%)$ & $3(30 \%)$ & 0 & 0.67 \\
$\begin{array}{l}\text { Two positive } \\
\text { signs }\end{array}$ & $6(85.7 \%)$ & $1(14.3 \%)$ & 0 & \\
$\begin{array}{l}\text { Three positive } \\
\text { signs }\end{array}$ & $3(60 \%)$ & $1(20 \%)$ & $1(20 \%)$ & \\
$\begin{array}{l}\text { Four positive } \\
\text { signs }\end{array}$ & $6(75 \%)$ & $1(12.5 \%)$ & $1(12.5 \%)$ & \\
\hline
\end{tabular}

Patients were assessed for the presence of pulmonary edema, pleural effusion, pedal edema, and ascites and were categorized based on the number of positive signs. Statistical test used: Fischer exact test.

Table 4: Association of VEXUS score with treatment

\begin{tabular}{lllll}
\hline Treatment & $\begin{array}{l}\text { VEXUS score } \\
\text { improving }\end{array}$ & $\begin{array}{l}\text { VEXUS score } \\
\text { no change }\end{array}$ & $\begin{array}{l}\text { VEXUS score } \\
\text { worsening }\end{array}$ & p value \\
\hline IV fluid yes & $1(33.3 \%)$ & $2(66.7 \%)$ & 0 & 0.10 \\
No & $21(77.8 \%)$ & $4(14.8 \%)$ & $2(7.4 \%)$ & \\
Dialysis yes & $1(50 \%)$ & $1(50 \%)$ & 0 & 0.53 \\
No & $21(75 \%)$ & $5(17.9 \%)$ & $2(7.1 \%)$ & \\
Inotropes yes & $10(83.3 \%)$ & $1(8.3 \%)$ & $1(8.3 \%)$ & 0.42 \\
No & $12(66.7 \%)$ & $5(27.8 \%)$ & $1(5.6 \%)$ & \\
Diuretics yes & $18(72 \%)$ & $5(20 \%)$ & $2(8 \%)$ & 0.80 \\
No & $4(80 \%)$ & $1(20 \%)$ & 0 & \\
\hline
\end{tabular}

Statistical test used the Fischer exact test

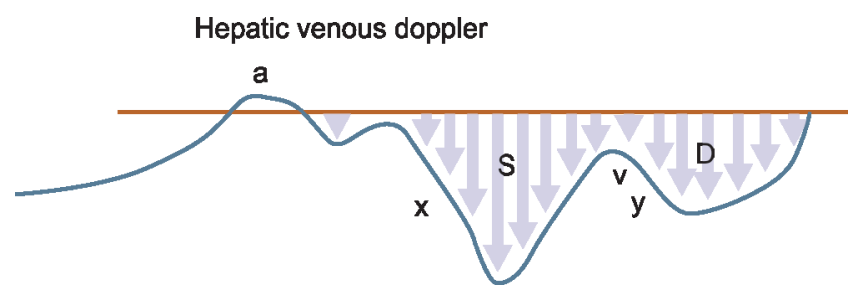

Fig. 2: Hepatic venous Doppler showing S wave and D wave (normal waveforms). The image has been taken from venous congestion chapter by Dr Philippe Rola after permission ${ }^{7}$

who had worsening of AKI showed either no change or worsening in VEXUS score correlating with the clinical picture. This finding shows that the dynamic trend of VEXUS grading correlated with the improving clinical condition.

Elevated CVP correlated with AKI as corroborated by a metaanalysis done by Chen et al. ${ }^{11}$ Central venous pressure did not normalize in those patients with AKI resolution showing CVP trend might not truly reflect the clinical picture because of its limitations, and possibly also because different patients may have different levels of tolerance of CVP, whereas Doppler flow markers may better reflect the severity of organ congestion and potential dysfunction. Peripheral signs of fluid overload did not correlate with AKI, which reemphasizes the fact that they might not truly reflect the intravascular volume status. ${ }^{5}$

Right heart dysfunction and VEXUS scoring did not have a statistically significant association suggesting that a high congestion score and its resolution reflecting resolving AKI was associated with venous fluid overload and not necessarily with cardiac dysfunction per se.

This study adds to the growing body of literature underscoring the critical importance of the venous side of the circulation, which, 


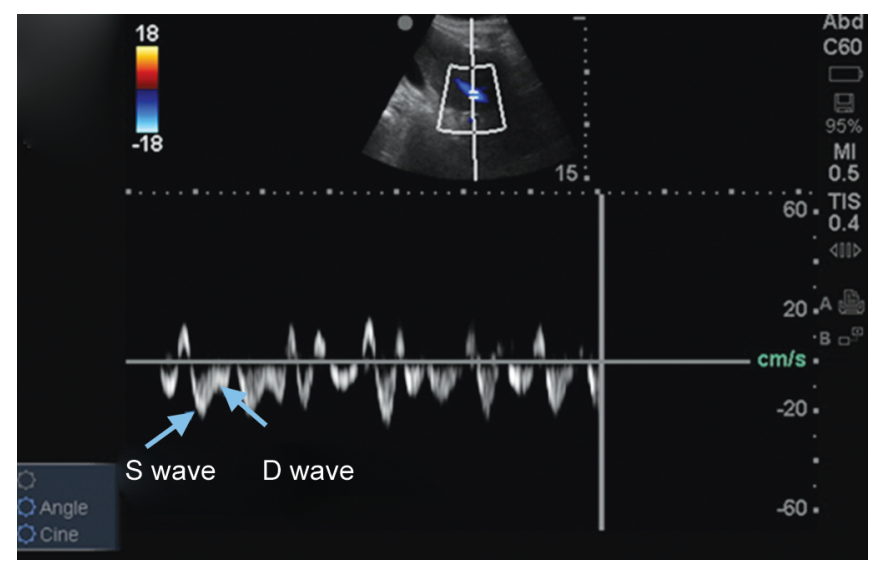

Fig. 3: Normal or grade $0 \mathrm{HD}$ with $\mathrm{S}$ wave more than D wave

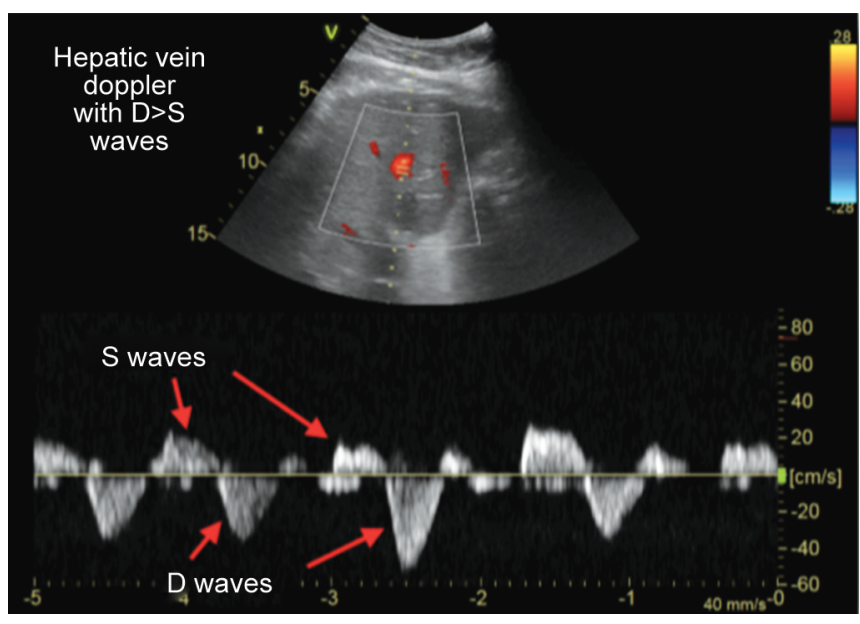

Fig. 4: Hepatic venous Doppler showing grade I congestion (the image has been taken from venous congestion chapter by Dr Philippe Rola after permission)
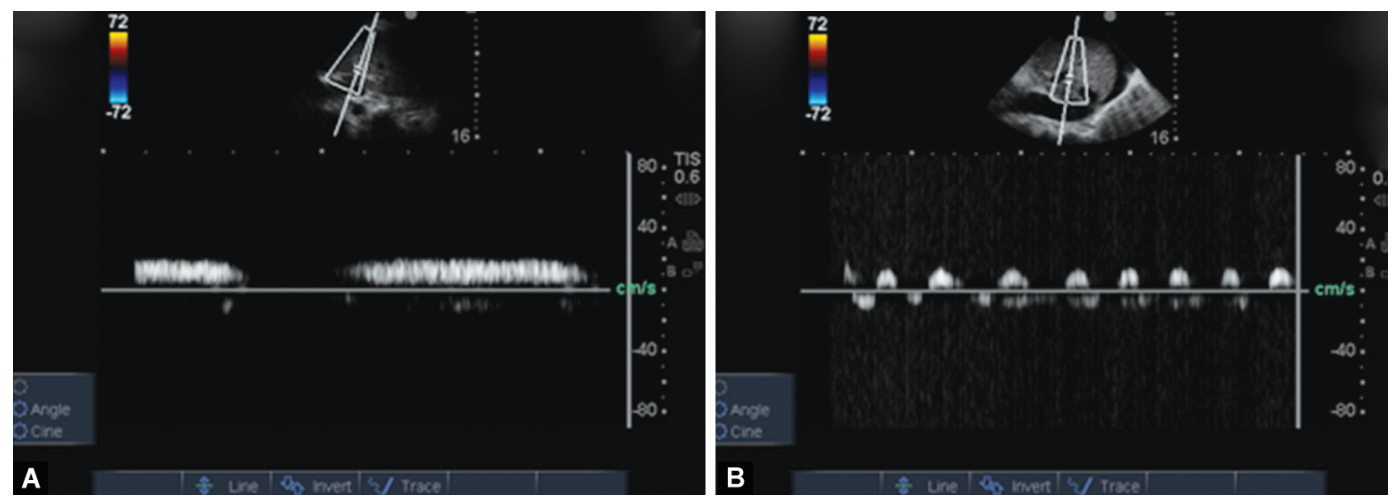

Figs 5A and B: (A) Monophasic portal vein grade 0; (B) Portal vein Doppler showing $100 \%$ pulsatility grade II PD

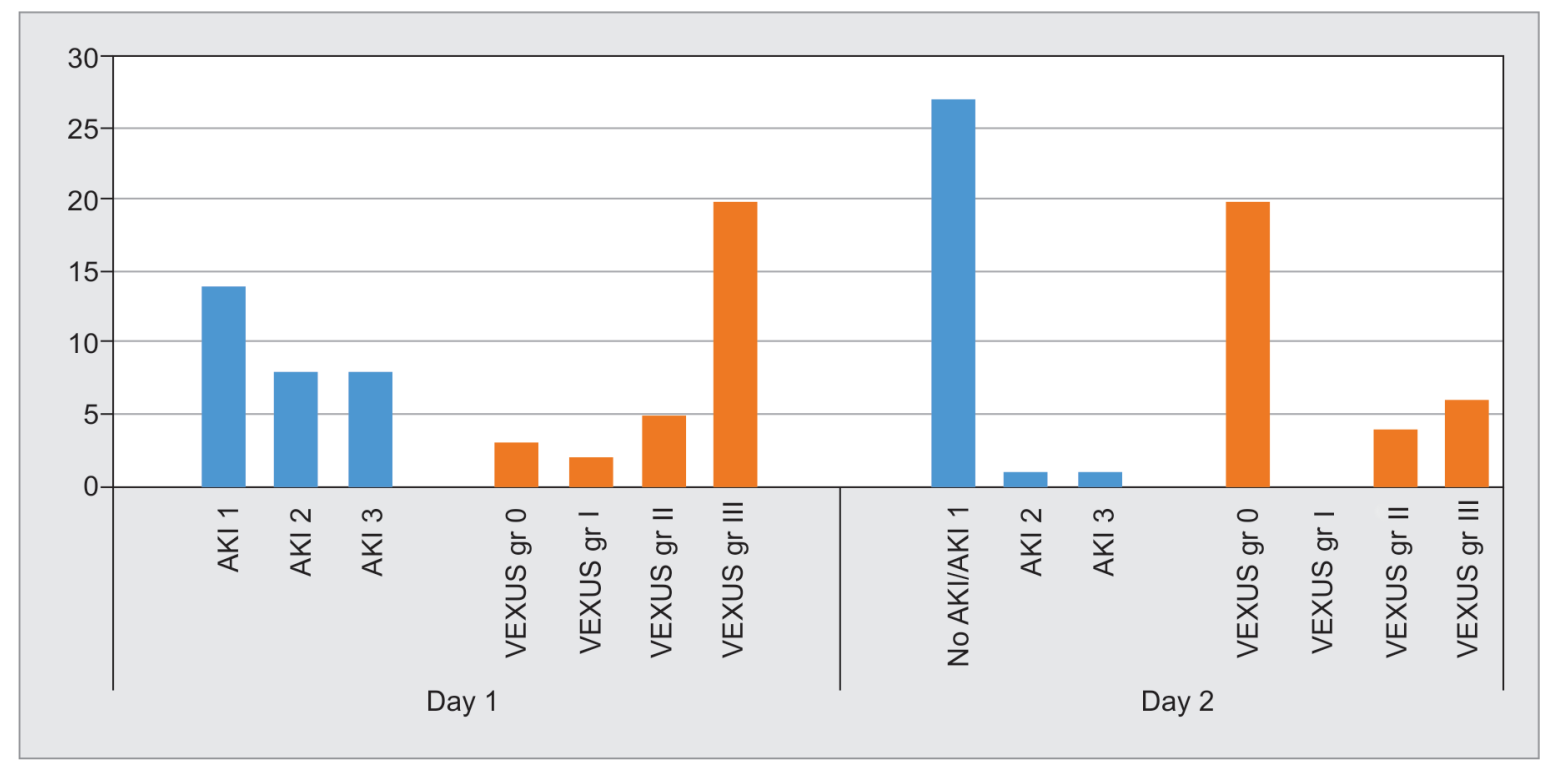

Fig. 6: Change in VEXUS grade with change in AKI stage

although not a novel concept, ${ }^{17}$ has been largely overlooked in favor of the arterial side ever since critical care has existed.

With the growing availability of point-of-care ultrasound (POCUS), dynamic flow parameters of physiology are becoming increasingly available to the clinician, many of which only require basic echographic competence. Along with the increasing prevalence of POCUS training in the core medical curriculum, this should change the landscape of clinical assessment in the next decade. 
Flowchart 1: Approach to AKI patient

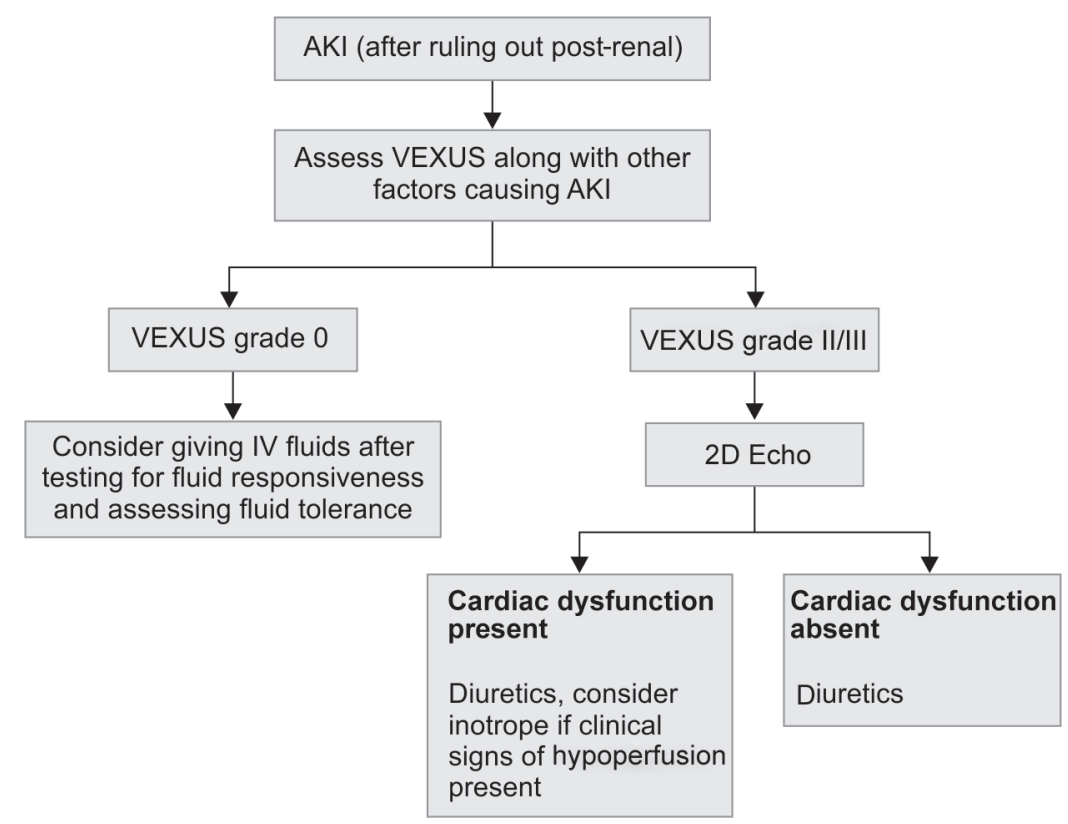

Given the prevalence of AKI in critically ill patients, fluid therapy is an important question. Unfortunately, clinicians today are confronted with contradictory data in the literature, with fluid resuscitation goals (SCCM reference of 8-12 CVP) ${ }^{18}$ and renal dysfunction indicators (Damman et al. up curve at 6-8 CVP of renal) being at odds with each other. ${ }^{19}$

Limitations of the study mainly include single-center study, small sample size, and observational design with no randomization of the patient. Additionally, as an observational study, it is hypothesis-generating, and a therapeutic direction cannot be widely implemented solely on its basis. Also, it is conducted in a specific group of patients with AKI that is cardiorenal syndrome.

Future directions: the VEXUS grading may be a useful tool in acute care and can be employed in various critical care scenarios:

- As a fluid stop and/or removal point in any fluid resuscitation (sepsis, hypovolemia)

- As a routine parameter in the evaluation of AKI to aid the clinical decision to add/increase the dose of diuretic and/or inotropic support

- In the critical phase of viral syndromes (severe dengue) to decide on fluids/diuretics

We propose a new flow chart as an initial approach to AKI subject to further testing by interventional clinical trials (Flowchart 1).

\section{CONCLUSION}

The above results show that a combined grading of IVC, hepatic vein, and portal vein might reliably demonstrate venous congestion and aid in the clinical decision to perform fluid removal. We conclude that bedside sonographic assessment of dynamic blood flow parameters is of great importance and warrants urgent further interventional trials.

\section{ReFERENCES}

1. Zhang L, Chen Z, Diao Y, Yang Y, Fu P. Associations of fluid overload with mortality and kidney recovery in patients with acute kidney injury: a systematic review and meta-analysis. J Crit Care 2015;30(4):860.e7-860.e13. DOI: 10.1016/j.jcrc.2015.03.025.

2. VaaraST,PettiläV,Kaukonen KM,BendelS, Korhonen AM,BellomoR, etal. Finnish acute kidney injury study group. the attributable mortality of acute kidney injury: a sequentially matched analysis. Crit Care Med 2014;42(4):878-885. DOI: 10.1097/CCM.0000000000000045.

3. Stevenson LW, Perloff JK. The limited reliability of physical signs for estimating hemodynamics in chronic heart failure. JAMA 1989;261(6):884-888. DOI: 10.1001/jama.1989.03420060100040.

4. Marik PE, Cavallazzi R. Does the central venous pressure predict fluid responsiveness? An updated meta-analysis and a plea for some common sense. Crit Care Med 2013;41(7):1774-1781. DOI: 10.1097/ CCM.0b013e31828a25fd.

5. Guinot PG, Abou-Arab O, Longrois D, Dupont H. Right ventricular systolic dysfunction and vena cava dilatation precede alteration of renal function in adult patients undergoing cardiac surgery: an observational study. Eur J Anaesthesiol 2015;32(8):535-542. DOI: 10.1097/EJA.0000000000000149.

6. De Vecchis R, Ariano C, Fusco A, Ciccarelli A, Cioppa C, Giasi A, et al. Ultrasound evaluation of the inferior vena cava collapsibility index in congestive heart failure patients treated with intravenous diuretics: new insights about its relationship with renal function: an observational study. Anadolu Kardiyol Derg 2012;12(5):391-400. DOI: 10.5152/akd.2012.121.

7. Rola P, Haycock K. Venous Congestion-Thinking Critical Care [Internet]. [cited April 2019]. Available from https://criticalcarethoughtsdotcom. files.wordpress.com/2019/04/venous-congestion chapter.pdf.

8. Denault AY, Beaubien-Souligny W, Elmi-Sarabi M, Eljaiek R, El-Hamamsy I, Lamarche $Y$, et al. Clinical significance of portal hypertension diagnosed with bedside ultrasound after cardiac surgery. Anesth Analg 2017;124(4):1109-1115. DOI: 10.1213/ ANE.0000000000001812.

9. Payen D, de Pont AC, Sakr Y, Spies C, Reinhart K, Vincent JL. A positive fluid balance is associated with a worse outcome in patients with acute renal failure. Crit Care 2008;12(3):R74. DOI: 10.1186/ cc6916.

10. Prowle JR, Bellomo R. Fluid administration and the kidney. Curr Opin Crit Care 2010;16(4):332-336. DOI: 10.1097/MCC.0b013e32833be90b.

11. Chen X, Wang X, Honore PM, Spapen HD, Liu D. Renal failure in critically ill patients, beware of applying (central venous) pressure 
on the kidney. Ann Intensive Care 2018;8(1):91. DOI: 10.1186/s13613018-0439-x.

12. Abu-Yousef MM. Normal and respiratory variations of the hepatic and portal venous duplex doppler waveforms with simultaneous electrocardiographic correlation. J Ultrasound Med 1992;11(6): 263-268. DOI: 10.7863/jum.1992.11.6.263.

13. Gallix BP, Taourel P, Dauzat M, Bruel JM, Lafortune M. Flow pulsatility in the portal venous system: a study of doppler sonography in healthy adults. AJR Am J Roentgenol 1997;169(1):141-144. DOI: 10.2214/ ajr.169.1.9207514.

14. Mehta RL, Kellum JA, Shah SV, Molitoris BA, Ronco C, Warnock DG, et al. Acute kidney injury network: report of an initiative to improve outcomes in acute kidney injury. Crit Care 2007;11(2):R31. DOI: 10.1186/ cc5713.

15. Lopes JA, Jorge S. The RIFLE and AKIN classifications for acute kidney injury: a critical and comprehensive review. Clin Kidney J 2013;6(1):8-14. DOI: 10.1093/ckj/sfs160.
16. Beaubien-Souligny W, Eljaiek R, Fortier A, Lamarche Y, Liszkowski M, Bouchard J, et al. The association between pulsatile portal flow and acute kidney injury after cardiac surgery: a retrospective cohort study. J Cardiothorac Vasc Anesth 2018;32(4):1780-1787. DOI: 10.1053/j. jvca.2017.11.030.

17. Maxwell MH, Breed ES, Schwartz IL. Renal venous pressure in chronic congestive heart failure. J Clin Invest 1950;29(3):342-348. DOI: 10.1172/JCl102263.

18. Rhodes A, Evans LE, Alhazzani W, Levy MM, Antonelli M, Ferrer $\mathrm{R}$, et al. Surviving sepsis campaign: international guidelines for management of sepsis and septic shock: 2016. Intensive Care Med 2017;43(3):304-377. DOI: 10.1007/s00134-017-4683-6.

19. Damman K, van Deursen VM, Navis G, Voors AA, van Veldhuisen $D J$, Hillege HL. Increased central venous pressure is associated with impaired renal function and mortality in a broad spectrum of patients with cardiovascular disease. J Am Coll Cardiol 2009;53(7):582-588. DOI: 10.1016/j.jacc.2008.08.080. 